\title{
Anion Stabilization in Electrostatic Environments
}

\section{Citation}

Olivares-Amaya, Roberto, Michael Stopa, Xavier Andrade, Mark A. Watson, and Alán AspuruGuzik. 2011. The Journal of Physical Chemistry Letters 2(7): 682-688.

\section{Published Version}

doi:10.1021/jz200120w

\section{Permanent link}

http://nrs.harvard.edu/urn-3:HUL.InstRepos:5344182

\section{Terms of Use}

This article was downloaded from Harvard University's DASH repository, and is made available under the terms and conditions applicable to Open Access Policy Articles, as set forth at http:// nrs.harvard.edu/urn-3:HUL.InstRepos:dash.current.terms-of-use\#OAP

\section{Share Your Story}

The Harvard community has made this article openly available.

Please share how this access benefits you. Submit a story.

Accessibility 


\title{
Anion Stabilization in Electrostatic Environments
}

\author{
Roberto Olivares-Amaya, ${ }^{\dagger}$ Michael Stopa, ${ }^{\ddagger}$ Xavier Andrade $,{ }^{\dagger}, \mathbb{I l}$ Mark A. Watson, ${ }^{\dagger}$ \\ and Alán Aspuru-Guzik*,†
}

Department of Chemistry and Chemical Biology, Harvard University, Cambridge, Massachusetts, 02138 , and Center for Nanoscale Systems, Harvard University, Cambridge, Massachusetts 02138

E-mail: aspuru@chemistry.harvard.edu

\footnotetext{
${ }^{*}$ To whom correspondence should be addressed

${ }^{\dagger}$ Department of Chemistry and Chemical Biology, Harvard University, Cambridge, Massachusetts, 02138

Center for Nanoscale Systems, Harvard University, Cambridge, Massachusetts 02138

"I Departamento de Física de Materiales, Facultad de Ciencias Químicas, UPV/EHU Centro Mixto CSIC-UPV/EHU and European Theoretical Spectroscopy Facility (ETSF), E-20018 San Sebastián, Spain
} 


\begin{abstract}
Excess charge stabilization of molecules in metallic environments is of particular importance for fields such as molecular electronics and surface chemistry. We study the energetics of benzene and its anion between two metallic plates. We observe that orientational effects are important at small inter-plate separation. This leads to benzene oriented perpendicular to the gates being more stable than the parallel case due to induced dipole effects. We find that the benzene anion, known for being unstable in the gas-phase, is stabilized by the plates at zero bias and an inter-plate distance of $21 \AA$. We also observe the effect of benzene under a voltage bias generated by the plates; under a negative bias, the anion becomes destabilized. We use the electron localization function to analyze the changes in electron density due to the bias. These findings suggest that image effects such as those present in nanoscale devices, are able to stabilize excess charge and should be important to consider when modeling molecular transport junctions and charge-transfer effects.
\end{abstract}

The stabilization of a transient electron in a molecular system by a complex nanoscale environment is relevant for the fields of molecular electronics and transport ${ }^{1-5}$ as well as to the understanding of molecular properties on surfaces such as in surface-enhanced Raman spectroscopy. ${ }^{6-13}$ A major challenge is that of understanding the interaction between extended metallic systems, such as surface metal gates or a nanoparticle, and an adsorbed molecule. From a theoretical standpoint, these two components are characterized by different lengthscales.

Differing approaches for handling this intrinsically multiscale problem have been taken. For example, several authors have focused on the molecular system. These efforts include the PCM ${ }^{14-17}$ and COSMO methodologies, ${ }^{18,19}$ where the metal surfaces or nanoparticles are simulated as cavity surfaces discretized into finite elements. The PCM technique has provided valuable insight into surface-enhancement processes. ${ }^{20-23}$ Recently, we have also modeled nanoscale systems using finite element and basis set methods. Our approach includes partitioning the Green's function into a contribution that is effectively the interaction of the particles in vacuum (obtaining the $1 / \mathbf{r}$ potential), and an additional term which is effectively due to the electrostatic environment (unpublished results). A parallel formulation has been developed by one of us for quantum dots. ${ }^{24-27}$ 
In this case, the system is determined by the finite-difference discretization of arbitrarily-shaped boundaries, where these lie at the edges of a calculation box. This treatment of gated systems has enabled the study of properties such as Coulomb blockade energetics, ${ }^{25}$ quantum-dots selfconsistent structures ${ }^{26}$ and more recently, Förster coupling in nanoparticle excitonic circuits. ${ }^{27}$

In this work we focus on molecular systems interacting with a nanoscale environment. As a representative example, we study the benzene anion. It is known that a single benzene molecule in the gas phase is unable to bind an excess electron as shown by a negative vertical electron affinity (VEA) of $-1.15 \mathrm{eV}^{28,29} \mathrm{VEA}$ is defined as the energy difference between the neutral species at its ground-state geometry and the anion species at the neutral geometry as shown in Eq. (1). We use atomic units throughout to represent all quantities, unless otherwise noted.

$$
\begin{aligned}
& \mathrm{VEA}=E_{\text {neutral }}\left(\mathbf{R}_{\text {neutral }}\right)-E_{\text {anion }}\left(\mathbf{R}_{\text {neutral }}\right) \\
& \mathrm{VDE}=E_{\text {neutral }}\left(\mathbf{R}_{\text {anion }}\right)-E_{\text {anion }}\left(\mathbf{R}_{\text {anion }}\right)
\end{aligned}
$$

Similar to the VEA, the vertical detachment energy (VDE), shown in Eq. (2) is defined as the energy difference between the neutral species at the anion's ground-state geometry and the anion at that same geometry. The VEA and VDE represent lower and upper bounds, respectively, of the electron affinity when the nuclear configuration is not significantly different in the anionic and neutral geometry. ${ }^{30-32}$ Specifically, Mitsui et al. ${ }^{33}$ observed the formation of negatively-charged benzene clusters $\left(\mathrm{C}_{6} \mathrm{H}_{6}\right)_{n}^{-}$. In the gas phase, the vertical detachment energy (VDE) is between 0.47 and $0.56 \mathrm{eV}$ for $n=53-124$ with an estimated value of $0.84 \mathrm{eV}$ when $n$ is extrapolated to infinity.

Why does a cluster of molecules bind an excess electron when a single molecule does not? One explanation is that the polarizability of the cluster is greater than the single molecule. An excess electron can therefore more readily induce a dipole in the cluster to which it can favorably interact. In a similar fashion, the polarizability of a local dielectric and conducting medium in a nanostructured system can also stabilize anionic states of molecules. 
In this Letter, we study the stability of the benzene anion in the proximity of a conductor. We approximate the metal surfaces as perfect conductors, which are grounded and can act as voltage sources. The arbitrariness of the boundaries allow us to explore geometric effects such as metallic tips. Variations from the parallel-plate setup treated in this paper are planned for future studies. We propose a simple method to determine the total energy of a system given an arbitrary electrostatic environment. We show that the benzene anion energy is lower than the neutral benzene energy in this environment. Subsequently, we describe the effect of a voltage bias on the system and explore the effects of the environment by studying the induced charge on the metal plate and the electronic localization function.

We place the molecule between two charged metallic plates, a geometry reminiscent of some single-molecule experiments. ${ }^{1,2}$ The total electrostatic energy of the molecule can then be given by two terms: the interaction of the charge density, $\rho(\mathbf{r})$, with the grounded potential $\phi^{\|}(\mathbf{r})$, and the interaction of the density with the potential generated due to the bias voltage at the plates, $\phi^{\mathrm{B}}(\mathbf{r})$. The grounded potential depends on the system's charge density: $\rho(\mathbf{r})=\rho_{\mathrm{ion}}(\mathbf{r})+\rho_{\mathrm{el}}(\mathbf{r})$ and is solved via the Poisson equation: $\nabla^{2} \phi^{\|}(\mathbf{r})=-4 \pi \rho(\mathbf{r})$; having the boundary condition: $\phi^{\|}\left(\mathbf{r}_{b}\right)=0 . \mathbf{r}_{b}$ represents the position of the plates, where the potential is set to zero. We use the dielectric constant of vacuum, though this can be easily extended to adopting a position-dependent dielectric function.

The bias potential is obtained by solving Laplace's equation, $\nabla^{2} \phi^{\mathrm{B}}(\mathbf{r})=0$, given the voltage bias, $\phi^{\mathrm{B}}\left(\mathbf{r}_{b}\right)=V_{b}$, in each of the gates. We use Laplace's equation as this potential is generated by charges outside the system. The expression of the total electrostatic system energy then becomes, $E_{e s}=\frac{1}{2} \int d \mathbf{r} \rho(\mathbf{r}) \phi^{\|}(\mathbf{r})+\int d \mathbf{r} \rho(\mathbf{r}) \phi^{\mathrm{B}}(\mathbf{r})$.

The plate energy also contributes to the total energy, but as we currently consider an open system, the plate energy essentially becomes the work associated with charging the metal plates up to a constant bias voltage. We return to discussing the plate energy below.

Given these non-trivial potentials, we can solve for the density in a self-consistent fashion by using the Kohn-Sham ${ }^{34,35}$ approach to density functional theory (DFT), where one solves a system 
of equations, $\left[-\frac{1}{2} \nabla^{2}+\phi^{\text {eff }}(\mathbf{r})\right] \chi_{i}(\mathbf{r})=\varepsilon_{i} \chi_{i}(\mathbf{r})$ where $\chi_{i}$ corresponds to a Kohn-Sham molecular orbital and $\varepsilon_{i}$ corresponds to its energy. In addition to the ionic and electronic potentials, contained in $\phi^{\|}(\mathbf{r}), \phi_{\text {eff }}$ includes the exchange-correlation potential, $\phi^{\mathrm{xc}}(\mathbf{r}): \phi^{\mathrm{eff}}=\phi^{\|}(\mathbf{r})+\phi^{\mathrm{B}}(\mathbf{r})+\phi^{\mathrm{xc}}(\mathbf{r})$. Finally, the density is determined by the occupied Kohn-Sham orbitals: $\rho_{\mathrm{el}}(\mathbf{r})=\sum_{i}\left|\chi_{i}(\mathbf{r})\right|^{2}$.

The internal energy $E_{\text {sys }}$ is given by the expression below. The superscripts in the potential terms represent the boundary condition and the subscripts represent the source of the potential,

$$
\begin{aligned}
E_{\mathrm{sys}}= & \sum_{i} \varepsilon_{i}-\frac{1}{2} \int d \mathbf{r} \rho_{\mathrm{el}}(\mathbf{r}) \phi_{\mathrm{el}}^{\|}(\mathbf{r})+ \\
& +\frac{1}{2} \int d \mathbf{r} \rho_{\mathrm{ion}}(\mathbf{r}) \phi_{\mathrm{ion}}^{\|}(\mathbf{r})+\int d \mathbf{r} \rho_{\mathrm{ion}} \phi^{\mathrm{B}}(\mathbf{r})+ \\
& +E^{\mathrm{xc}}\left[\rho_{\mathrm{el}}\right]-\int d \mathbf{r} \phi^{\mathrm{xc}}(\mathbf{r}) \rho_{\mathrm{el}}(\mathbf{r})
\end{aligned}
$$

Here $\varepsilon_{i}$ are the Kohn-Sham eigenvalues. $\phi_{\mathrm{el}}$ and $\phi_{\text {ion }}$ are the electronic and ionic potentials that act on the electronic and ionic densities: $\rho_{\mathrm{el}}$ and $\rho_{\mathrm{ion}}$, respectively. $E^{\mathrm{xc}}\left[\rho_{\mathrm{el}}\right]$ is the exchangecorrelation energy. Note that the sum over Kohn-Sham eigenvalues in Eq. (3) contains implicitly the electrostatic interaction of the electrons: $\frac{1}{2} \int d \mathbf{r} \rho_{\mathrm{el}}(\mathbf{r}) \phi_{\mathrm{el}}^{\|}(\mathbf{r})+\int d \mathbf{r} \rho_{\mathrm{el}}(\mathbf{r}) \phi_{\mathrm{el}}^{\mathrm{B}}(\mathbf{r})$.

In free-space, the ion potential is given by $\phi_{\text {ion }}^{\circ}(\mathbf{r})$ and the ion-ion interaction, $\frac{1}{2} \int d \mathbf{r} \rho_{\text {ion }}(\mathbf{r}) \phi_{\text {ion }}^{\circ}(\mathbf{r})$, is substituted by the sum of the $i$ th-ion interacting with all other $j$ th-ions: $\sum_{i} \sum_{i>j} \frac{q_{i} q_{j}}{\left|R_{i}-R_{j}\right|}$. In arbitrary electrostatic conditions, besides considering the interaction between the $i$ th-ion and the potential generated by the $j$ th ion: $\sum_{i} \sum_{i>j} \int d \mathbf{r} \rho_{\text {ion }, i}(\mathbf{r}) \phi_{\text {ion }, j}^{\|}(\mathbf{r})$, the interaction given by an ion with the metal plates is an important energetic component. ${ }^{36}$ This can be rationalized in physical terms as the interaction of each ion with its mirror images. This contribution is an attractive one and is fully considered in our calculations (as detailed in the Supporting Information) and is included in Eq. (3).

Since we assume the constant potential generators are attached to power supplies (or ground lines), the system is effectively open and the work done by the environment must be included in the total free energy $F$. The energy of the charges on the $m$ metal surfaces is given by the plate 
energy,

$$
E_{g}=\frac{1}{2} \sum_{m} Q_{m} V_{m},
$$

where $Q_{m}$ represents the surface charge and $V_{m}$ the applied voltage at the gates. $Q_{m}$ is obtained as the normal derivative of the total electrostatic potential, $\phi(\mathbf{r})=\phi^{\|}(\mathbf{r})+\phi^{\mathrm{B}}(\mathbf{r})$, at each of the surfaces: $Q_{m}=\left.\frac{-1}{4 \pi} \frac{\partial \phi(\mathbf{r})}{\partial n}\right|_{\mathbf{r}=\mathbf{r}_{b}}$. The standard expression for the work done by batteries in electrical circuit analysis is,

$$
\begin{aligned}
W & =-\sum_{m} \int d t I_{m}(t) V_{m}(t) \\
& =-\sum_{m} Q_{m} V_{m}
\end{aligned}
$$

where $V_{m}(t)$ is the time-dependent applied voltage, and $I_{m}(t)$ is the current to or from the power supply, for the $m$ th gate respectively. Eq. (5) results from the assumption that the voltage is maintained arbitrarily close to the power supply potential throughout any charge rearrangement processes that induce a flow of current to or from the system.

Taking into account the additional energy terms of Eq. (4) and Eq. (5), the final expression for the total free energy of the system becomes:

$$
\begin{aligned}
F & =E_{\mathrm{sys}}+\frac{1}{2} \sum_{m} Q_{m} V_{m}-\sum_{m} \int d t V_{m}(t) I_{m}(t) \\
& =E_{\mathrm{sys}}-\frac{1}{2} \sum_{m} Q_{m} V_{m} .
\end{aligned}
$$

For all results presented in this work, Eq. (6) is what we will refer to as the energy of the system.

We study a model system composed of two parallel-infinite plates separated along the z-axis. The gates are assumed to be connected to power supplies which can be set to arbitrary voltages. The plates confine a benzene molecule oriented either parallel or perpendicular to the metallic plates, shown in Figure 1a and Figure 1b, respectively.

We performed all calculations using Octopus, ${ }^{37}$ a finite-difference real-space DFT code, which we modified to include the electrostatic boundary conditions. We modeled the system using the 


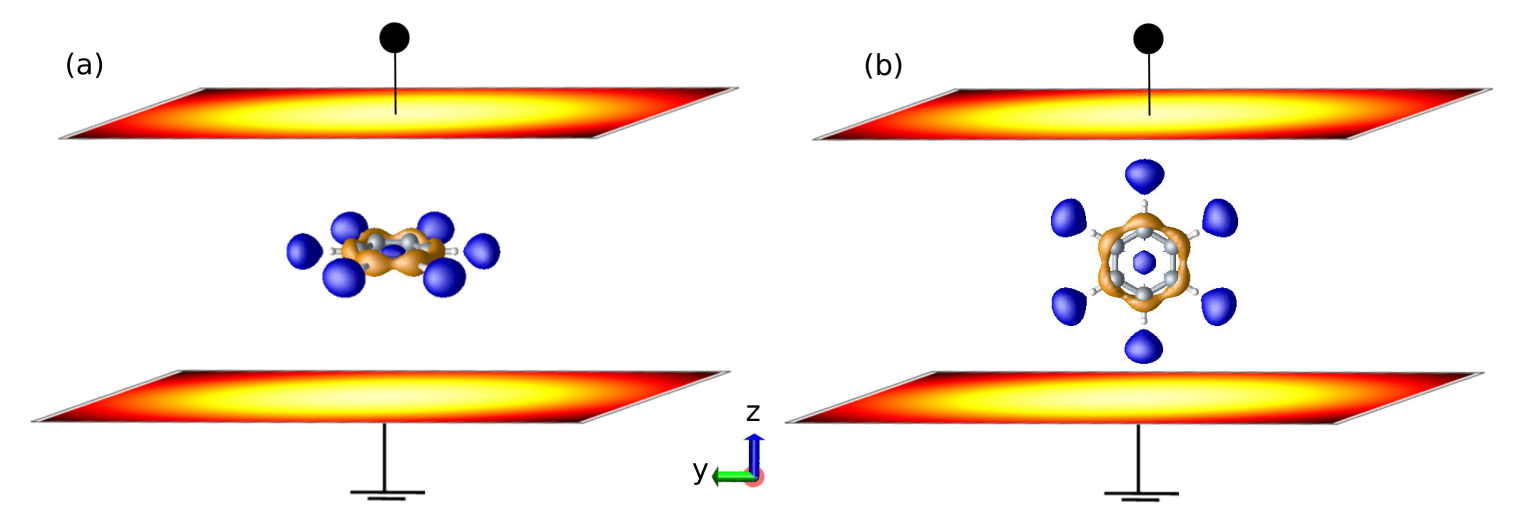

Figure 1: Two metallic plates acting on a molecule. Two metal plates measuring $6 \mathrm{~nm}$ on each side separated by $2.1 \mathrm{~nm}$ (figure not to scale) confine a molecular system either parallel (a) or perpendicular (b) to the plates. We present the induced charge (See Figure $4 \mathrm{a}-\mathrm{b}$ ). In between the plates, we show the highest occupied molecular orbital (HOMO) of the benzene anion.

Perdew-Burke-Ernzerhof (PBE) functional. ${ }^{38-40}$ The interaction between valence electrons and the cores were modeled using Troullier-Martins pseudopotentials. ${ }^{41}$ Method details are available on section $\mathrm{S} 1$ of the Supporting Information.

We first look at the total energy of benzene with respect to the plate separation, while keeping the molecule centered and the gates grounded. The results are plotted in Figure 2. When the interplate distance is $13 \AA$, the perpendicular orientation is $3.3 \mathrm{eV}$ lower in energy than the parallel orientation. This implies that the molecule centered between the plates will tend to rotate toward the perpendicular orientation. This effect results from the charge induced, by the molecule, in the metal plates and is thus a type of Casimir effect. ${ }^{42-44}$

The conformational stabilization of benzene with respect to the plates can be understood in the context of image charges. In the perpendicular case, the molecule is closer to the metal plates, as seen in Figure 1. The image charge solution gives an opposite charge on the plates to each of the charges present inside the plates. We therefore expect that proximity to the plate translates as an additional source of energy stabilization. As the plate separation increases, at $40 \AA$ plate distance, both orientations are within $0.1 \mathrm{eV}$ of each other.

Next, we explore a bias sweep by varying the potential on the bottom plate (see Figure 1) and keeping the top grounded. Neutral benzene presents a parabolic dependence of the energy with respect to the voltage bias centered around $0.0 \mathrm{~V}$. A positive or negative bias affects the energy 


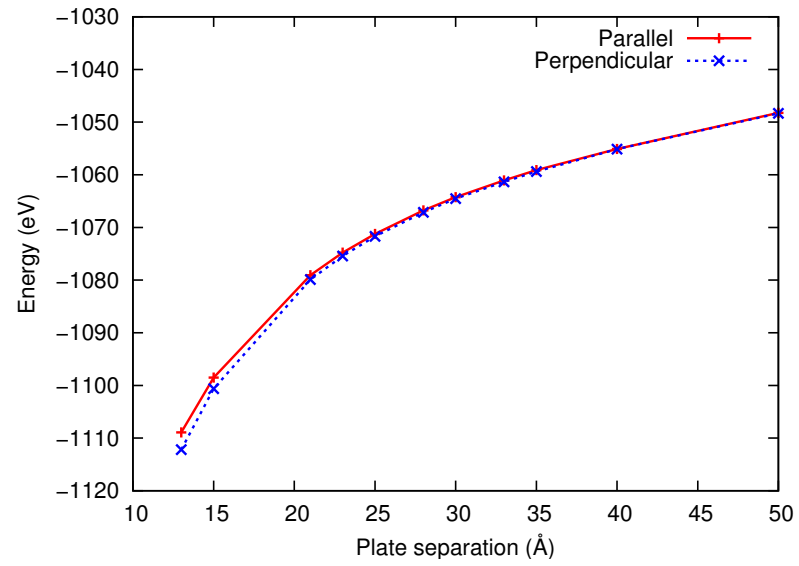

Figure 2: Benzene total energy with respect to plate separation. The total energy of benzene at parallel and perpendicular orientations with respect to the metal plate distance separation. The latter orientation is higher in energy at lower plate separations, but as the plates become farther apart, these energies converge and the orientational effects of energy disappear.

similarly, as shown in Figure 3 where the change of energy takes a parabolic shape.

The benzene anion experiences stabilization due to the image charges in the metallic plates of an overall charged system. This leads to a stabilization of the excess electron in benzene. When the plates are grounded (i. e. no bias), we obtain a total energy which is $0.4 \mathrm{eV}$ lower than the neutral system for both orientations. In this case, the maximum is not at $0.0 \mathrm{~V}$, but rather is shifted to a potential of approximately $-0.3 \mathrm{~V}$ for both orientations. At this potential, the excess electron has some electron density interacting with the bottom plate. The energy curves are not symmetric due to the excess electron, which causes a different response with positive and negative biases. It stabilizes under a positive bias more readily than under a negative one due to the excess negative charge. As the voltage bias increases (becomes more positive), the slope for the total energy of the anion becomes steeper than in the case of the negative bias. Finally, for large negative potential, we find that the neutral system becomes more stable than the anion at a bias of $-1.0 \mathrm{eV}$ and of $-1.1 \mathrm{eV}$ for the parallel and perpendicular cases, respectively as is seen in Figure 3.

We continue by looking at the induced charge on the metal plates due to its energetic relevance. With the anion oriented parallel to the plates the induced charge shows a round shape, while it shows an oval shape when the molecule is oriented perpendicular to the plates (along the xz-plane) as seen in Figure 4a and Figure 4b, respectively. The induced charge on the molecule parallel 

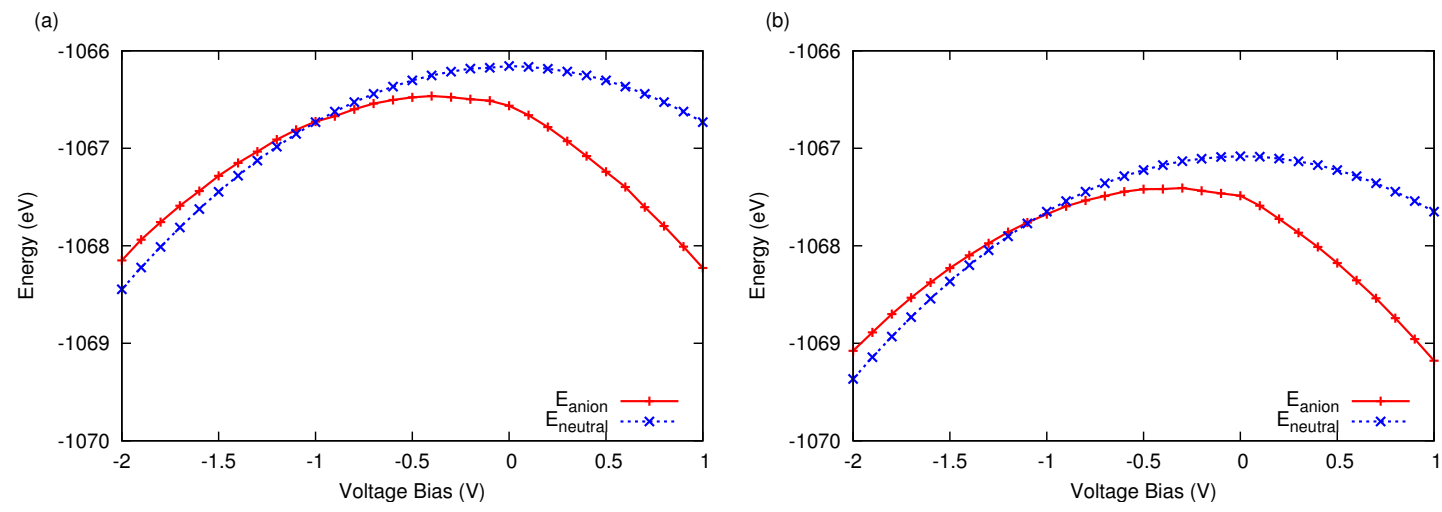

Figure 3: Benzene anion energy and its neutral counterpart. Energy between the benzene anion and its neutral counterpart when the molecule is oriented parallel (a) and perpendicular (b) to the plate and an inter-plate distance of $21 \AA$. At zero voltage bias, the energy of the anion is less than the neutral molecule. Adding a negative voltage bias moves the neutral case towards higher stability with respect to the anion.

to the plates is relatively homogeneous across the boundary, while in the perpendicular case, the induced charge is concentrated in the center and has a more pronounced response. In both cases it is positive, indicating that the excess electron is screened by the plates. The induced charge is therefore helpful to identify the areas of increased electron density along system boundaries.

We plot the highest-occupied molecular orbital (HOMO) of the molecule with plates grounded and at a bias of $-0.1 \mathrm{~V}$ as shown in Figure $5 \mathrm{a}$ and Figure $5 \mathrm{~b}$, respectively. The comparison of these orbitals suggests an increased polarizability of the excess electron as it diffuses when applying a bias in the electrostatic environment.

As shown in Figure 5c, the anion HOMO energy at zero bias is $-0.20 \mathrm{eV}$. There is a change of HOMO energy as we apply the voltage bias; decreasing in energy as the bias is increased. Changes in HOMO and LUMO levels influence charge transport calculations and studies of these changes are planned as a future area of exploration.

One can also look at the interaction betwen the molecule and the plates as the system interacting with its image charges; the latter being responsible for the anion stabilization. Specifically, the image charges provide a screened potential, different than the $1 / \mathbf{r}$ potential present in vacuum which allows the excess electron to interact more strongly with the ions. To further understand the interaction of the electrons and ions of the system, we use the electron localization function (ELF) 

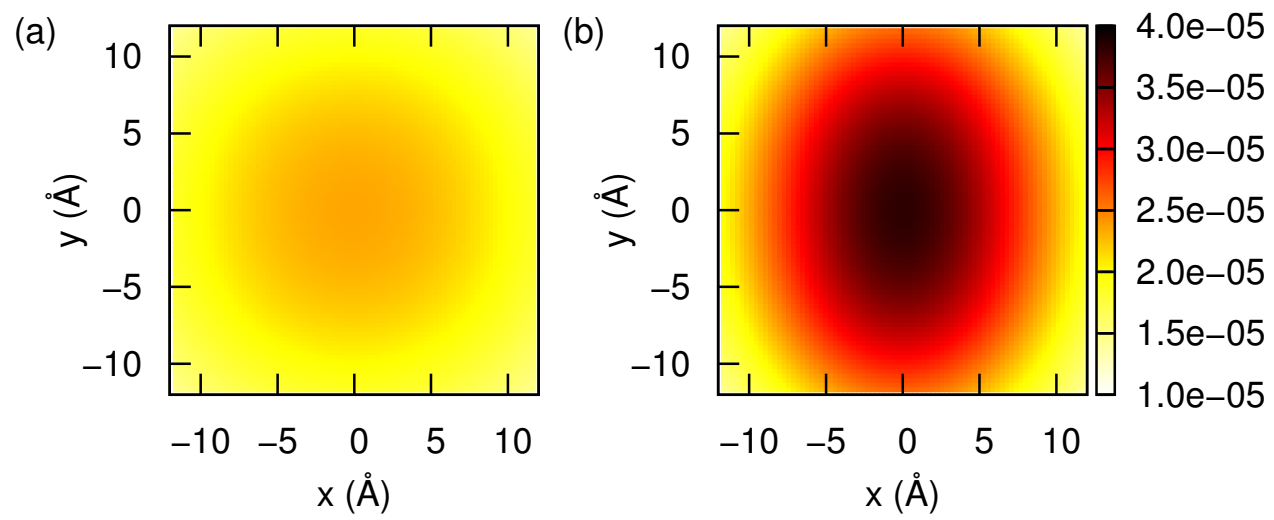

Figure 4: Induced charge (a.u.) at bottom plate caused by benzene anion at different orientations. (a) Parallel to the plates. (b) Perpendicular to the plates.

developed by Becke and Edgecombe. ${ }^{45}$ Description of the ELF can be found in section $\mathrm{S} 2$ of the Supporting Information.

There are clear differences in the case of the molecule in free-space and under these electrostatic conditions. In Figure 6 we compare the ELF of the benzene anion between the metal plates to its free-space form. In order to highlight this, we plot the ELF difference: $\triangle \mathrm{ELF}=\mathrm{ELF}^{\mathrm{I}}-\mathrm{ELF}^{\circ}$, where the $\approx$ and $\circ$ superscripts denote the metallic plates and free-space, respectively. 2D $\Delta \mathrm{ELF}$ plots (showing the $\mathrm{yz}$ plane at $\mathrm{x}=0$ ) for different bias potentials are shown in Figure 6 . Figure $6 \mathrm{~b}$ at $\mathrm{V}=0.0$ shows crescent-shaped regions at $z= \pm 4 \AA$ with a negative difference (blue). A negative value of $\triangle E L F$ represents regions where, in the metallic plates case, the excess electron is able to penetrate closer to the ions. The difference of the ELF diagrams between benzene anion in freespace and grounded with the metal plates, shown in Figure 6b, signifies that the excess electron lies closer to the nuclei in the latter case. Effectively, this causes the excess electron to be more strongly bound in this environment by $0.4 \mathrm{eV}$.

As we decrease the voltage bias, we observe the depletion of the excess electron as a negative voltage bias is added to the bottom plate of the system, as shown in Figure 6b. In vacuum, this section is completely dominated by the excess electron. As we add a negative bias, this electron 
(a)

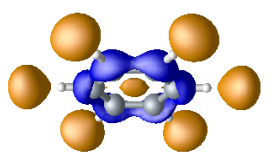

(b)

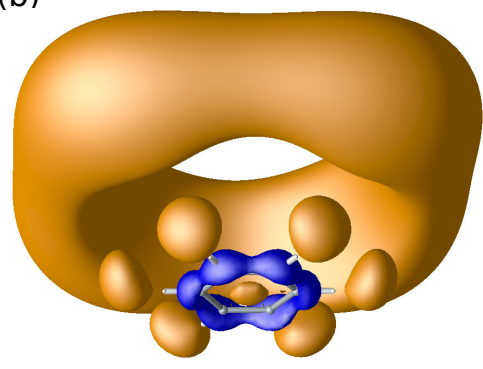

(c)

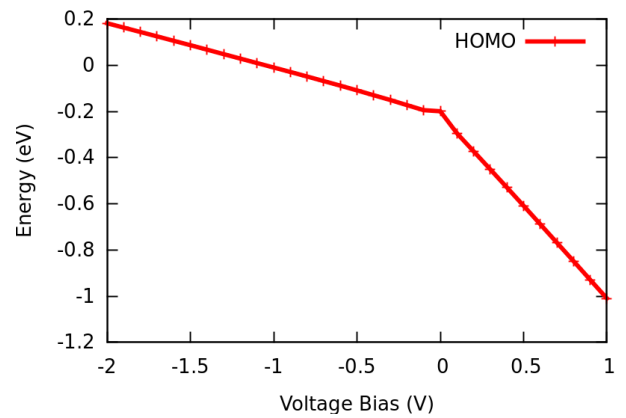

Figure 5: Highest occupied molecular orbital (HOMO) of the benzene anion (a) Benzene anion HOMO under no bias under the electrostatic environment. (b) Benzene anion HOMO with a $-0.1 \mathrm{~V}$ bias shows an increased localization of the orbital away from the bias. (c) HOMO eigenvalue at different voltage bias.

will tend to avoid the bottom-half area. At the same time, we obtain a negative difference in ELF at positive values of $z$. As we decrease the bias, the bottom region becomes depleted of the excess electron. Similarly, the top area acquires more electron density, but the $\Delta$ ELF in this area remains at -0.5 ; as the excess electron is already dominating in this region.

The plate distance separation calculations show the relevance of image charge effects due to the plates. The energetic contribution due to the metal plates become an essential contribution to understand orientational effects. For instance, at small inter-plate distances, the energy differences are in the range of several $\mathrm{eV}$. We notice that each ion or electron will also interact with the images of the other charged particles of the system. It can be shown by geometric arguments that the interaction with the plate(s) is attractive for a system with any number of charges when the plates are grounded.

In the case of the benzene anion, these image charges cause the excess electron to stabilize, with respect to its neutral counterpart. When applying a voltage bias, the system's total energy becomes part of an energetic interplay between the decrease in energy of the eigenvalues (particularly the HOMO energy, as seen in Figure 5c), the electrostatic energies and the plate energy.

Choi et al. have explored the molecular orbital shift of benzene under a electrostatic field in the order of $1 \mathrm{VA}^{-1} \cdot{ }^{46,47}$ The bias induced in the metallic plates produces also an electric field. In order to create such fields in this confined environment, we require a voltage bias of $21 \mathrm{~V}$. In 

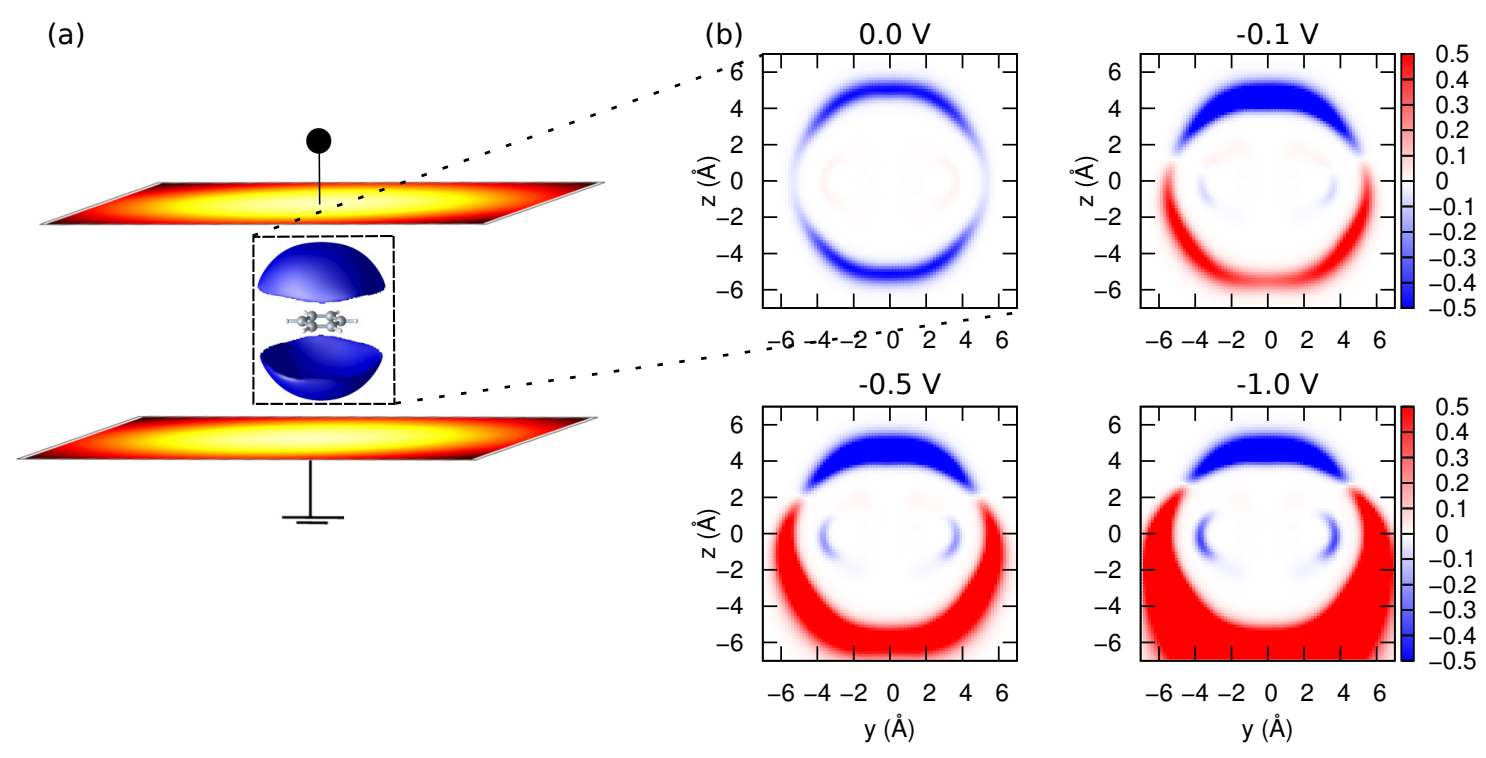

Figure 6: Difference of metallic-environment ELF: $\triangle \mathbf{E L F}=\mathbf{E L F}^{ \pm}-\mathbf{E L F}^{\circ}$, where $=$ and $\circ$ represent the metallic plates and free-space, respectively, at different voltage biases of $\beta$-spin polarization contains the excess electron. (a) represents $\triangle E L F$. The dotted region represents the area plotted in (b). We plot the $\mathrm{yz}$-plane at $\mathrm{x}=0$. (b) shows $\triangle \mathrm{ELF}$ for four different voltages. In $\mathrm{V}=0.0$, the diagram shows an area (blue) where the excess electron dominates closer to the molecular region, and edges (red) where the electrons are more delocalized than the free-space case. As we decrease the bias to more negative bias the bottom region becomes more red (positive) as the excess electron begins to localize itself upwards, due to the electrostatic repulsion with the plates.

the case of the benzene anion, less voltage is needed to observe effects similar to those studied by Choi et al., such as molecular orbital tuning, as shown in Figure 5c. We observe the effect of a small field $\sim 0.1 \mathrm{V \AA}^{-1}$ as it causes the excess electron distribution to move away from the plate with negative voltage.

The work of Mitsui et al. ${ }^{33}$ showed the stabilization of an excess charge in benzene clusters of 53-124 molecules. We observe that a similar effect takes place in an metallic environment: the VEA becomes positive, and thus an excess charge in benzene is stabilized. Other conjugated molecules, such as polycyclic aromatic hydrocarbons, have a systematically decreasing VEA ${ }^{48}$ as they increase in number of aromatic cycles.

The effects presented here by simulating a metallic environment with electrostatic boundary condition reveal the leading role of the image charge effect for stabilizing excess charge in molecular systems. This work shows a straightforward way of considering electron confinement in a 
perfect conductor system.

The use of more geometrically-complex boundary conditions will allow us to obtain an even more realistic environment of the molecule interacting with an extended metallic system. Furthermore, controlling the molecule in this environment could greatly enhance the system's spectroscopic properties such as is the case for surface-enhanced and tip-enhanced Raman scattering.

The implementation of this algorithm in a versatile and widely-available software package such as Octopus will allow for studies of time-dependent properties of the molecules and also will facilitate the simulations of higher-order physics such as many-body effects with metallic boundary conditions in the near future.

\section{Acknowledgements}

This work was supported by the Defense Advanced Research Project Agency under Contract No. FA9550-08-1-0285 and Defense Threat Reduction Agency under Contract No. HDTRA1-10-10046. This work was partially conducted while R.O.A. was a Giorgio Ruffolo Fellow in the Sustainability Science Program at Harvard University's Center for International Development. X.A. acknowledges support from the European Community e-I3 ETSF project (Contract No. 211956) and "Grupos Consolidados UPV/EHU del Gobierno Vasco" (IT-319-07). M.A.W. gratefully acknowledges NSF PHY-0835713 for financial support. Computations in this paper were run on the Odyssey cluster supported by the FAS Research Computing Group. We also thank the US National Nanotechnology Infrastructure Network Computation project for technical support.

The authors wish to thank Prof. Hongkun Park for originally suggesting this problem, as well as P. Rebentrost, Drs. D. Rappoport, and S. Saikin and Prof. Angel Rubio for stimulating discussions.

\section{Supporting Information Available}

Method details, ions in electrostatic environments, geometries and energetic contributions. This material is available free of charge via the Internet at http://pubs.acs.org/. 


\section{References}

(1) Reed, M.; Zhou, C.; Muller, C.; Burgin, T.; Tour, J. Conductance of a Molecular Junction. Science 1997, 278, 252-254.

(2) Liang, W.; Shores, M. P.; Bockrath, M.; Long, J. R.; Park, H. Kondo Resonance in a SingleMolecule Transistor. Nature 2002, 417, 725-729.

(3) Nitzan, A.; Ratner, M. A. Electron Transport in Molecular Wire Junctions. Science 2003, $300,1384-1389$.

(4) Solomon, G. C.; Herrmann, C.; Hansen, T.; Mujica, V.; Ratner, M. A. Exploring Local Currents in Molecular Junctions. Nature Chem. 2010, 2, 223-228.

(5) Dell'Angela, M.; Kladnik, G.; Cossaro, A.; Verdini, A.; Kamenetska, M.; Tamblyn, I.; Quek, S. Y.; Neaton, J. B.; Cvetko, D.; Morgante, A.; Venkataraman, L. Relating Energy Level Alignment and Amine-Linked Single Molecule Junction Conductance. Nano Lett. 2010, 10, 2470-2474.

(6) Fleischmann, M.; Hendra, P. J.; McQuillan, A. J. Raman Spectra of Pyridine Adsorbed at a Silver Electrode. Chem. Phys. Lett. 1974, 26, 163-166.

(7) Schatz, G. C. Theoretical Studies of Surface Enhanced Raman Scattering. Acc. Chem. Res. 1984, 17, 370-376.

(8) Moskovits, M. Surface-Enhanced Spectroscopy. Rev. Mod. Phys. 1985, 57, 783-826.

(9) Corni, S.; Tomasi, J. Surface Enhanced Raman Scattering from a Single Molecule Adsorbed on a Metal Particle Aggregate: A Theoretical Study. J. Chem. Phys. 2002, 116, 1156-1164.

(10) Jensen, L.; Aikens, C. M.; Schatz, G. C. Electronic Structure Methods for Studying SurfaceEnhanced Raman Scattering. Chem. Soc. Rev. 2008, 37, 1061-73. 
(11) Dieringer, J. A.; Wustholz, K. L.; Masiello, D. J.; Camden, J. P.; Kleinman, S. L.; Schatz, G. C.; Van Duyne, R. P. Surface-Enhanced Raman Excitation Spectroscopy of a Single Rhodamine 6G Molecule. J. Am. Chem. Soc. 2009, 131, 849-854.

(12) Saikin, S. K.; Olivares-Amaya, R.; Rappoport, D.; Stopa, M.; Aspuru-Guzik, A. On the Chemical Bonding Effects in the Raman Response: Benzenethiol Adsorbed on Silver Clusters. Phys. Chem. Chem. Phys. 2009, 11, 9401-11.

(13) Saikin, S. K.; Chu, Y.; Rappoport, D.; Crozier, K. B.; Aspuru-Guzik, A. Separation of Electromagnetic and Chemical Contributions to Surface-Enhanced Raman Spectra on Nanoengineered Plasmonic Substrates. J. Phys. Chem. Lett. 2010, 1, 2740-2746.

(14) Tomasi, J.; Persico, M. Molecular Interactions in Solution: An Overview of Methods Based on Continuous Distributions of the Solvent. Chem. Rev. 1994, 94, 2027-2094.

(15) Tomasi, J.; Mennucci, B.; Cammi, R. Quantum Mechanical Continuum Solvation Models. Chem. Rev. 2005, 105, 2999-3093.

(16) Corni, S.; Tomasi, J. From Metal Nanoparticles and Nanoparticles Aggregates with Continuum Models. Top. Appl. Phys. 2006, 124, 105-123.

(17) Mennucci, B. Continuum Solvation Models: What Else Can We Learn from Them? J. Phys. Chem. Lett. 2010, 1, 1666-1674.

(18) Klamt, A.; Schüürmann, G. COSMO: a new Approach to Dielectric Screening in Solvents with Explicit Expressions for the Screening Energy and its Gradient. J. Chem. Soc., Perkin Trans. 2 1993, 799-805.

(19) Klamt, A. Conductor-like Screening Model for Real Solvents: A New Approach to the Quantitative Calculation of Solvation Phenomena. J. Phys. Chem. 1995, 99, 2224-2235.

(20) Corni, S.; Tomasi, J. Enhanced Response Properties of a Chromophore Physisorbed on a Metal Particle. J. Chem. Phys. 2001, 114, 3739-3751. 
(21) Vukovic, S.; Corni, S.; Mennucci, B. Fluorescence Enhancement of Chromophores Close to Metal Nanoparticles. Optimal Setup Revealed by the Polarizable Continuum Model. J. Phys. Chem. C 2009, 113, 121-133.

(22) Muñoz-Losa, A.; Vukovic, S.; Corni, S.; Mennucci, B. Nonplasmonic Metal Particles as Excitation Energy Transfer Acceptors: an Unexpected Efficiency Revealed by Quantum Mechanics. J. Phys. Chem. C 2009, 113, 16364-16370.

(23) Sánchez-González, A.; Muñoz Losa, A.; Vukovic, S.; Corni, S.; Mennucci, B. Quantum Mechanical Approach to Solvent Effects on the Optical Properties of Metal Nanoparticles and Their Efficiency As Excitation Energy Transfer Acceptors. J. Phys. Chem. C 2010, 114, $1553-1561$.

(24) Stopa, M. Coulomb Oscillation Amplitudes and Semiconductor Quantum-Dot SelfConsistent Level Structure. Phys. Rev. B 1993, 48, 18340-18343.

(25) Stopa, M.; Aoyagi, Y.; Sugano, T. Multiple-Capacitance Magnetic-Field-Dependent coulomb Blockade Energetics. Phys. Rev. B 1995, 51, 5494-5497.

(26) Stopa, M. Quantum Dot Self-Consistent Electronic Structure and the Coulomb Blockade. Phys. Rev. B 1996, 54, 13767-13783.

(27) Rebentrost, P.; Stopa, M.; Aspuru-Guzik, A. Förster Coupling in Nanoparticle Excitonic Circuits. Nano Lett. 2010, 10, 2849-56.

(28) Boness, M.; Larkin, I.; Hasted, J.; Moore, L. Virtual Negative Ion Spectra of Hydrocarbons. Chem. Phys. Lett. 1967, 1, 292-294.

(29) Nenner, I.; Schulz, G. Temporary Negative Ions and Electron Affinities of Benzene and Nheterocyclic Molecules: Pyridine, Pyridazine, Pyrimidine, Pyrazine, and s-triazine. J. Chem. Phys. 1975, 62, 1747-1758.

(30) Simons, J. Molecular Anions. J. Phys. Chem. A 2008, 112, 6401-6511. 
(31) Rienstra-Kiracofe, J. C.; Tschumper, G. S.; Schaefer, H. F.; Nandi, S.; Ellison, G. B. Atomic and Molecular Electron Affinities: Photoelectron Experiments and Theoretical Computations. Chem. Rev. 2002, 102, 231-82.

(32) Kronik, L.; Fromherz, R.; Ko, E.; Ganteför, G.; Chelikowsky, J. R. Highest Electron Affinity as a Predictor of Cluster Anion Structures. Nature Mater. 2002, 1, 49-53.

(33) Mitsui, M.; Nakajima, A.; Kaya, K.; Even, U. Mass Spectra and Photoelectron Spectroscopy of Negatively Charged Benzene Clusters, (benzene) $)_{n}^{-}(\mathrm{n}=53-124)$. J. Chem. Phys. 2001, 115, $5707-5710$.

(34) Hohenberg, P.; Kohn, W. Inhomogeneous Electron Gas. Phys. Rev. 1964, 136, B864-B871.

(35) Kohn, W.; Sham, L. Self-Consistent Equations Including Exchange and Correlation Effects. Phys. Rev. 1965, 140, A1133-A1138.

(36) Jackson, J. D. Classical Electrodynamics Third Edition, 3rd ed.; Wiley, 1998.

(37) Castro, A.; Appel, H.; Oliveira, M.; Rozzi, C. A.; Andrade, X.; Lorenzen, F.; Marques, M. A. L.; Gross, E. K. U.; Rubio, A. Octopus: a Tool for the Application of Time-Dependent Density Functional Theory. Phys. Stat. Sol. B 2006, 243, 2465-2488.

(38) Perdew, J.; Burke, K.; Ernzerhof, M. Generalized Gradient Approximation Made Simple. Phys. Rev. Lett. 1996, 77, 3865-3868.

(39) Perdew, J.; Burke, K.; Ernzerhof, M. Generalized Gradient Approximation Made Simple [Phys. Rev. Lett. 77, 3865 (1996)]. Phys. Rev. Lett. 1997, 78, 1396(E).

(40) Zhang, Y.; Yang, W. Comment on "Generalized Gradient Approximation Made Simple". Phys. Rev. Lett. 1998, 80, 890.

(41) Troullier, N.; Martins, J. Efficient Pseudopotentials for Plane-Wave Calculations. Phys. Rev. B 1991, 43, 1993-2006. 
(42) Milonni, P. W.; Shih, M.-L. Casimir Forces. Contemp. Phys. 1992, 33, 313-322.

(43) Noguez, C.; Román-Velázquez, C. E.; Esquivel-Sirvent, R.; Villarreal, C. High-Multipolar Effects on the Casimir Force: The Non-Retarded Limit. Europhys. Lett. 2004, 67, 191-197.

(44) Capasso, F.; Munday, J. N.; Iannuzzi, D.; Chan, H. B. Casimir Forces and Quantum Electrodynamical Torques: Physics and Nanomechanics. IEEE J. Sel. Top. Quantum Electron. 2007, $13,400-414$.

(45) Becke, A. D.; Edgecombe, K. E. A Simple Measure of Electron Localization in Atomic and Molecular Systems. J. Chem. Phys. 1990, 92, 5397-5403.

(46) Choi, Y. C.; Kim, W. Y.; Park, K.-S.; Tarakeshwar, P.; Kim, K. S.; Kim, T.-S.; Lee, J. Y. Role of Molecular Orbitals of the Benzene in Electronic Nanodevices. J. Chem. Phys. 2005, 122, 094706.

(47) Kim, W. Y.; Kim, K. S. Tuning Molecular Orbitals in Molecular Electronics and Spintronics. Acc. Chem. Res. 2010, 43, 111-120.

(48) Rienstra-Kiracofe, J.; Barden, C.; Brown, S.; Schaefer III, H. Electron Affinities of Polycyclic Aromatic Hydrocarbons. J. Phys. Chem. A 2001, 105, 524-528. 


\section{Graphical TOC Entry}

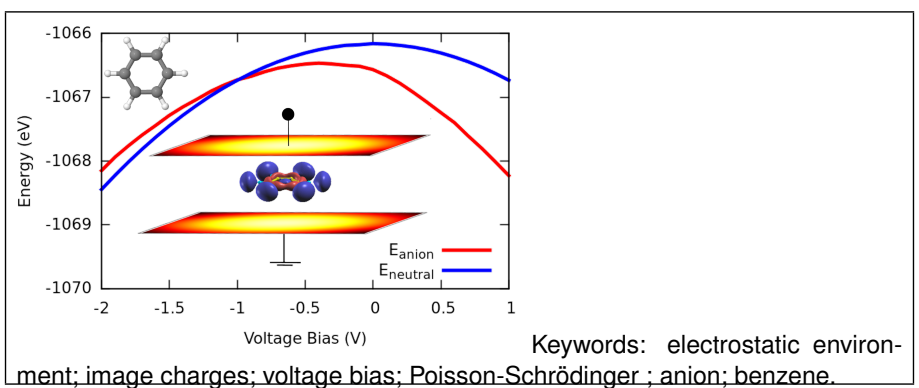

